\title{
HEAVY SUBGRAPH PAIRS FOR TRACEABILITY OF BLOCK-CHAINS
}

\author{
Binlong Li ${ }^{a, b 1}$, Hajo Broersma ${ }^{b}$ \\ AND \\ SHENGGUI ZHANG ${ }^{a 2}$ \\ ${ }^{a}$ Department of Applied Mathematics \\ Northwestern Polytechnical University \\ Xi'an, Shaanxi 710072, P.R. China \\ ${ }^{b}$ Faculty of EEMCS, University of Twente \\ P.O. Box 217, 7500 AE Enschede, The Netherlands \\ e-mail: h.j.broersma@utwente.nl
}

\begin{abstract}
A graph is called traceable if it contains a Hamilton path, i.e., a path containing all its vertices. Let $G$ be a graph on $n$ vertices. We say that an induced subgraph of $G$ is $o_{-1}$-heavy if it contains two nonadjacent vertices which satisfy an Ore-type degree condition for traceability, i.e., with degree sum at least $n-1 \mathrm{in} G$. A block-chain is a graph whose block graph is a path, i.e., it is either a $P_{1}, P_{2}$, or a 2-connected graph, or a graph with at least one cut vertex and exactly two end-blocks. Obviously, every traceable graph is a block-chain, but the reverse does not hold. In this paper we characterize all the pairs of connected $o_{-1}$-heavy graphs that guarantee traceability of block-chains. Our main result is a common extension of earlier work on degree sum conditions, forbidden subgraph conditions and heavy subgraph conditions for traceability.
\end{abstract}

Keywords: $o_{-1}$-heavy subgraph, block-chain traceable graph, Ore-type condition, forbidden subgraph.

2010 Mathematics Subject Classification: 05C45, 05C38, $05 \mathrm{C} 07$.

\section{REFERENCES}

\footnotetext{
${ }^{1}$ Supported by the Doctorate Foundation of Northwestern Polytechnical University (No. cx201202).

${ }^{2}$ Supported by NSFC (No. 11271300).
} 
[1] P. Bedrossian, G. Chen, and R.H. Schelp, A generalization of Fan's condition for hamiltonicity, pancyclicity, and hamiltonian connectedness, Discrete Math. 115 (1993) 39-50. doi:10.1016/0012-365X(93)90476-A

[2] J.A. Bondy and U.S.R. Murty, Graph Theory, Springer Graduate Texts in Mathematics 244 (2008).

[3] H.J. Broersma, Z. Ryjáček and I. Schiermeyer, Dirac's minimum degree condition restricted to claws, Discrete Math. 167/168 (1997) 155-166. doi:10.1016/S0012-365X(96)00224-5

[4] H.J. Broersma and H.J. Veldman, Restrictions on induced subgraphs ensuring hamiltonicity or pancyclicity of $K_{1,3}$-free graphs, in: R. Bodendiek (Ed.) Contemporary Methods in Graph Theory, (BI Wissenschaftsverlag, Mannheim-Wien-Zfirich, 1990) 181-194.

[5] R. Čada, Degree conditions on induced claws, Discrete Math. 308 (2008) 5622-5631. doi:10.1016/j.disc.2007.10.026

[6] D. Duffus, R.J. Gould and M.S. Jacobson, Forbidden subgraphs and the hamiltonian theme, in: The Theory and Applications of Graphs (Kalamazoo, Mich. 1980, Wiley, New York, 1981) 297-316.

[7] R.J. Faudree and R.J. Gould, Characterizing forbidden pairs for Hamiltonian properties, Discrete Math. 173 (1997) 45-60. doi:10.1016/S0012-365X(96)00147-1

[8] H. Fleischner, The square of every two-connected graph is hamiltonian, J. Combin. Theory (B) 16 (1974) 29-34. doi:10.1016/0095-8956(74)90091-4

[9] B. Li, H.J. Broersma and S. Zhang, Forbidden subgraph pairs for traceability of block-chains, Electron. J. Graph Theory Appl. 1 (2013) 1-10.

[10] B. Li, Z. Ryjáček, Y. Wang and S. Zhang, Pairs of heavy subgraphs for Hamiltonicity of 2-connected graphs, SIAM J. Discrete Math. 26 (2012) 1088-1103. doi: $10.1137 / 11084786 \mathrm{X}$

[11] B. Li and S. Zhang, On traceability of claw-o-1-heavy graphs (2013). arXiv:1303.0991v1

Received 11 April 2012

Revised 14 March 2013

Accepted 14 March 2013 